\title{
Construction of Simulation for Adsorption Process Visualization
}

\author{
Byeongtae Ahn
}

\begin{abstract}
Recently, with the development of biotechnology, much interest has been focused on chromatographic adsorption processes for separation of biomolecules such as proteins, which are important. The efficiency of the adsorption process was improved by visualizing the data by simulation. The system visualized numerical results according to adsorption process modeling easily and efficiently. It is possible to predict the expected result even if the numerical data is inputted without going through the conventional experiment. The purification process of biological and chemical processes may involve several stages of chromatographic separation processes. The system visualizes the results of the simulation according to the adsorption process modeling or makes it appear as a curve graph. Therefore, in this paper, we design and implement a simulation system for adsorption process modeling. The currently developed simulation visualization system is a visualization of the result values that are driven by the existing engine, so future work will enable the engine to operate in the system itself.
\end{abstract}

Keywords : Adsorption, Imulation, Modeling, Optimization, Visualization

\section{INTRODUCTION}

Recently, the key basis of information technology is shifting from the convergence between technologies and products to the convergence between IT and industries, contributing to sophistication and productivity of industries and creating noble technologies and industries. U-campus in the ubiquitous society is changing, along with the rapid development of IT, to the direction that allows users to build network and communicate at anytime anywhere through wireless, internet laptop, PDA (personal digital assistant), PMP (portable multimedia player), and smart phones[1]. Instead, U-campus should facilitate interactions among space, users and services considering physical space, user behaviors, and digital environment [2]. To that end, various researching and development activities are underway to build a ubiquitous computing environment. Already in many advanced countries, establishing an internet campus utilizing ubiquitous computing technologies has been underway[3].

Currently, the severity of air pollution in urban areas including Seoul is increasing, and various policies and technologies are being developed to improve them. In the

Revised Manuscript Received on July 22, 2019.

Byeongtae Ahn, Liberal \& Arts College, Anyang University, Anyang-shi, South Korea. Email: ahnbt@anyang.ac.kr case of large cities, most of the air pollution originates from mobile pollution sources such as automobiles. In addition, strengthening the emission allowance standards for incinerators and pollutants around urban areas and the emission trading system will be implemented. In the case of urban areas, melting incineration is expected to be applied in a cleaner garbage disposal technique in the future. From this point of view, it is expected that the development of post-treatment technology will be a very important part, and the application of hybrid SNCR-SCR process can be considered as a technical alternative and it will contribute to the improvement of air quality [2].

Considering the market size of 1 trillion won, which is based on the nitrogen oxides emitted from fixed sources, it is expected that more than 100 billion won of NOx abatement market will be formed annually [3]. In this market, it is expected that more economical and efficient technology demand will increase, and it will be possible to reduce the investment cost and improve the removal efficiency through the hybrid of selective catalytic reduction (SCR) and selective non-catalytic reduction (SNCR)[4]. The successful development of the hybrid SNCR-SCR technology will create demand for more than 50 high-quality human resources annually. Assuming that $10 \%$ of the market size of 100 billion is applied to the hybrid process, . Also, it is expected to revitalize the domestic market by overcoming the current technical difficulties [5].

Currently, there are various sources of nitrogen oxides such as incinerators, medium and large boilers, thermal power plants, cement kilns, and combined power plants. Domestic and overseas demand for nitrogen oxides reduction technology Of the total. In terms of removal efficiency, the selective catalytic reduction process is the most excellent, but the use of noble metal catalysts has a disadvantage of high initial investment [6]. In the case of existing facilities, there is not enough space to install the selective catalytic reduction facility, and a large amount of facilities must be built, which causes a lot of cost. In this paper, we design and implement a simulation system for the adsorption process modeling that can accelerate the process design for process development from the laboratory scale to the commercial scale and present an efficient experimental plan [7]. In this system, the result of the solute concentration is shown by a curve graph or visualized by the concentration change video. In Section 2 of this paper, we describe the mathematical operation method for modeling the adsorption process. In Section 3, we design a simulation system for modeling the adsorption process. In Section 4, simulation system design is

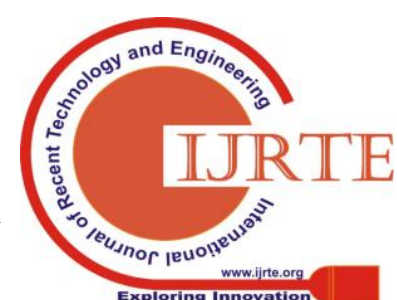


implemented. Section 5 presents conclusions and future work.

\section{HIGH PERFORMANCE ADSORPTION PROCESS MODELING}

Adsorption process models are classified into equilibrium models and non - equilibrium models. Both models consist of high performance adsorption process modeling by the following equation, and the results are derived according to the solute concentration. The resulting value is visualized as a curved graph and video of the system.

$$
\begin{aligned}
& \text { linear model(option_flag }=1400 \text { or 1410) }: n_{i}{ }^{*}=K_{i} C_{i} \text { (1) } \\
& \text { normal langmuir(option_flag=1310): } n_{i}^{x}=\frac{a_{i} C_{Q_{1}}}{1+b_{i} C_{1}} \\
& \text { competitive Lnagmuir(option_flag=1320) : } \\
& n_{\mathrm{i}}^{x}=\frac{\mathbb{a}_{\mathrm{i}} C_{\mathrm{R}}}{1+\sum_{\mathbb{E}}^{\mathrm{BH}}} \\
& \text { competitive bi-Langmuir type(option_flag=1330) : }
\end{aligned}
$$

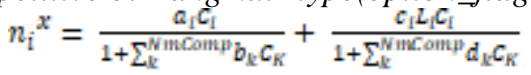

$$
\begin{aligned}
& \text { modified Lnagmuir type(option_flag=1340) : } \\
& n_{\mathrm{i}}^{x}=\frac{\mathbb{a}_{\mathrm{i}} K_{\mathrm{i}} C_{\mathrm{R}}}{1+\sum_{\mathrm{L}}}+c_{\mathrm{i}} C_{\mathrm{i}}
\end{aligned}
$$

polynomials(option_flag=1350): $n_{i}^{x}=a_{0 i}+\sum_{n-1} a_{\ni} C_{i}^{n}$

$$
\begin{aligned}
& \text { Ching-model(option_flag=1360) : } \\
& n_{i}^{x}=K_{i} C_{I}+a_{i} C_{i}^{[m+1)}+b_{i j} C_{j}^{m_{i}} C_{i}
\end{aligned}
$$

mass action kinetic model(option_flag=1370) : Lim et al.(2005).

User-defined model(option_flag=1300)

In the above equation, ni $*$ denotes the equilibrium concentration of solute in the pore of adsorbed particles, and $\mathrm{Ci}$ denotes the concentration of solute in the fluidized bed. Other symbols are model constants that are empirically derived or predicted. In this paper, we have chosen to designate a unique number according to each adsorption process. In order to optimize the simulation of the adsorption process, it is necessary to determine the column porosity, the axial dispersion coefficient $d$ value and the mass transfer coefficient $\mathrm{k}$. The resultant value is derived by using the completed input data according to each formula.

\section{SIMULATION PLATFORM SYSTEM DESIGN}

In Section 3, we propose a design method for building a system using the equations presented in section 2 .

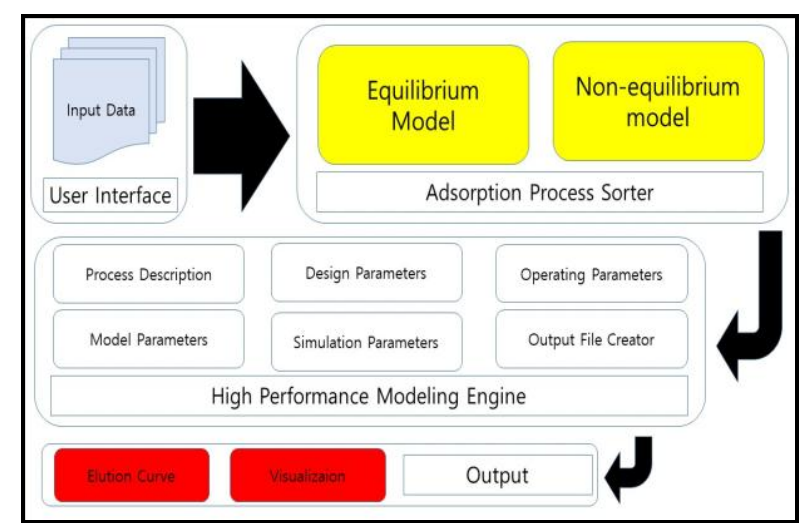

Fig. 1. Simulation System Structure

Fig. 1 shows the overall structure of a high performance adsorption process simulation system. In the user interface, input values required for simulation of the adsorption process modeling are written for each step. After inputting the data in 7 stages, it is classified into equilibrium model or non-equilibrium model based on the data input from the adsorption process classifier and sent to the high-performance modeling engine [8]. The high-performance modeling engine transfers the 7-step total data from the user interface to the process description item.

The process description section classifies the process model. The classified model is transferred to the design parameter, and the data value is inputted according to the number of the columns, the length and the inner diameter. In the operation parameter, the user inputs the number of ingredients of the raw material among the data entered in the step 7 and automatically aligns them. In the model parameter, the isothermal absorption coefficient according to the number of components is input and the result value is transmitted to the simulation parameter. The simulation parameters are derived by inputting the number of partial differential equations induced in each component and outputting the result of the adsorption process modeling to a file. The derived values are generated and produced as 4 result files through the output file generator. Each created file is played back as a curved graph or visualized video at the final output stage [9].

\section{IMPLEMENTATION OF SIMULATION PLATFORM SYSTEM}

In Section 4, we implemented a simulation system for modeling the adsorption process. We implemented the system using Visual Basic 2010 on Windows 2012 server. The formula proposed in Chapter 2 is constructed using FORTRAN program and it is visualized as curve graph and moving image at the last output part by receiving input data at each stage in the actual user interface[10].

As such u-CUPS has reflected characteristics of 3 different campuses, as a customized information system and improved efficiency of work by directly supporting of integrated information system. 


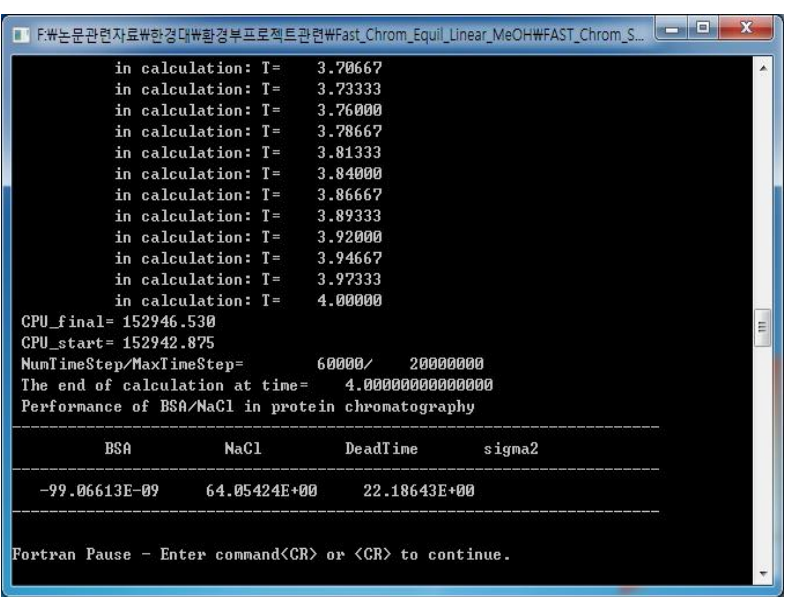

Fig. 2. Simulation Number Result Value

Fig. 2 shows the result of running the FORTRAN program implemented using the formula presented in Chapter 2 by applying the input data of step 7 . The actual processing time of the central processing unit takes $4000 \mathrm{~ms}$, and the result of the chromatic analysis is shown for each item. The result value for each item appears as four files in the input result tab.

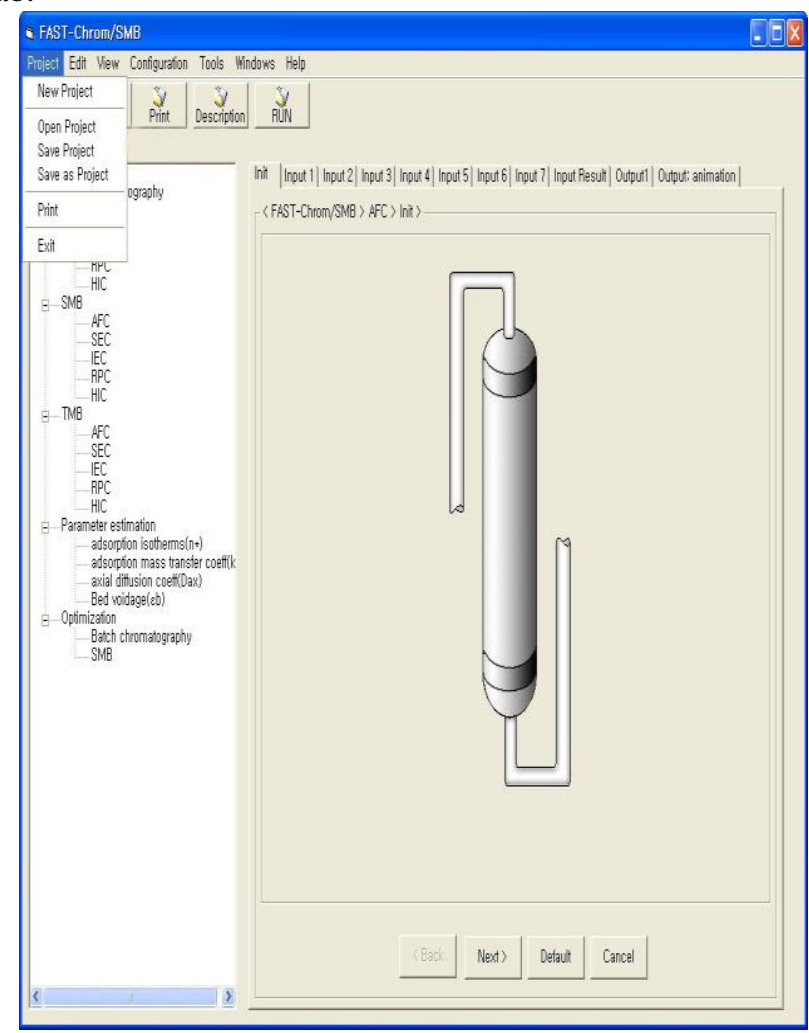

Fig. 3. Adsorption Modeling System Interface

Fig. 3 shows the initial screen of the adsorption process modeling system. The menu consists of a total of 7 main menus. When you click a new project in the project item, the system starts up. In the initial screen, input values for each item are classified into 7 levels as shown in the right screen. After inputting each item, go to the next item, the engine applying the formula given in Chapter 2 will be activated and the result value will be returned.

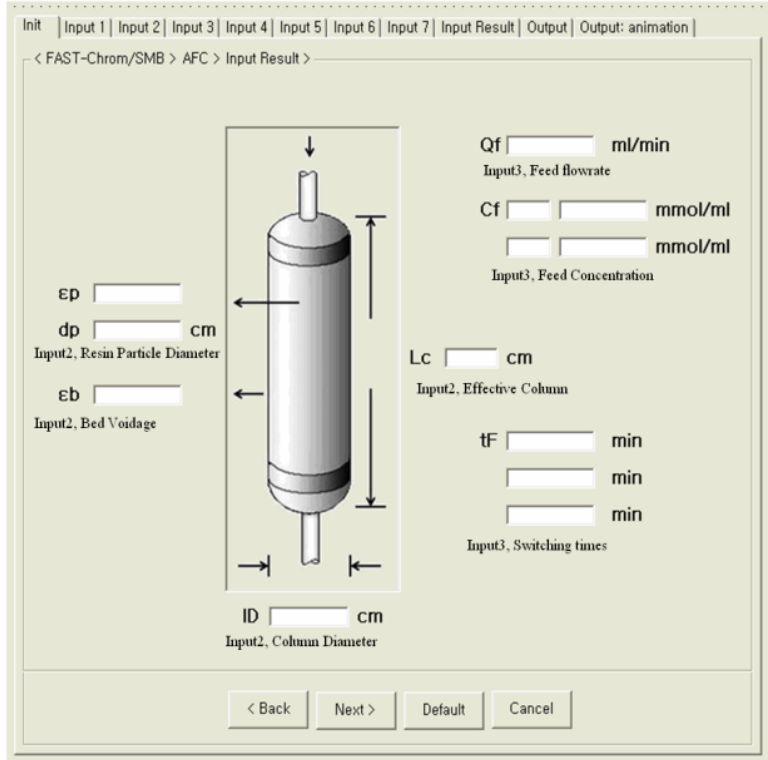

Fig. 4. Initial Project Driving

Fig. 4 shows the initial screen when starting a new project. For each item, enter the information value of the basic environmental variable. For more detailed items, describe the condition variable values in the input step of 7 stages in total. The Default button provides default environment variable values. The Cancel button is used to initialize or re-enter the input variable value.

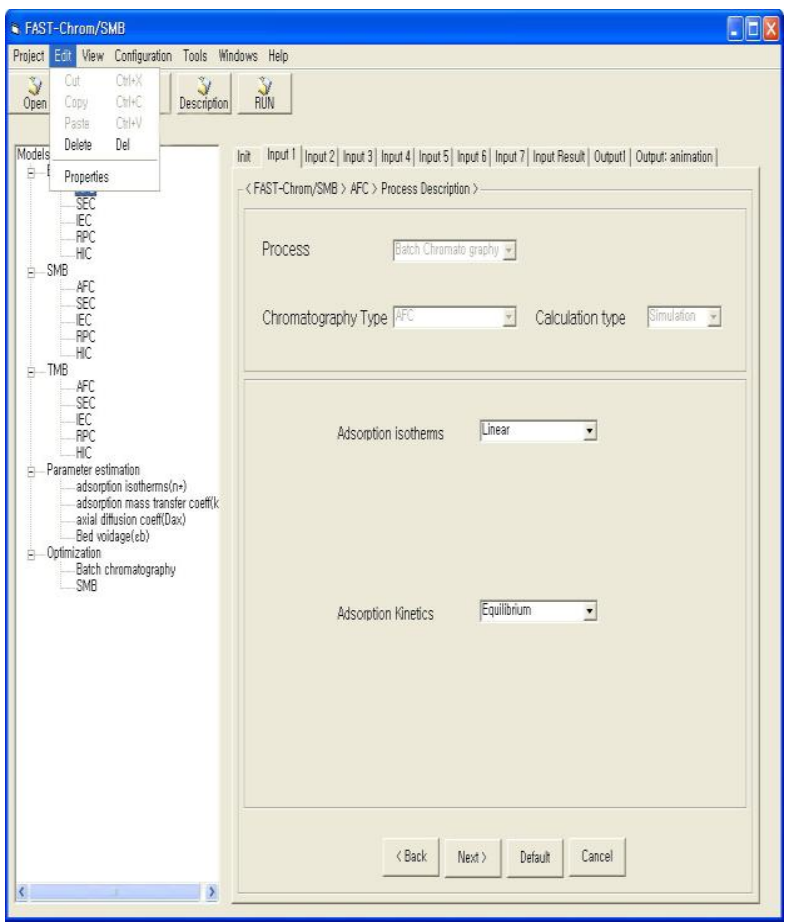

Fig. 5. Itemized Input Step

Fig. 5 shows the first step of input for each item when the project is started. In the first step, the element for each item is selected from the list on the left screen based on the initial environment information value. The other items designate the item desired by the user among the predetermined value items and switch to the next step. After inputting the following items for each step and selecting

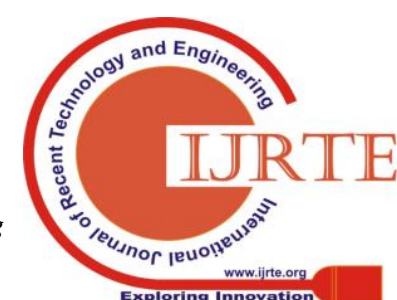


the Input Result Value tab, four result files are generated.

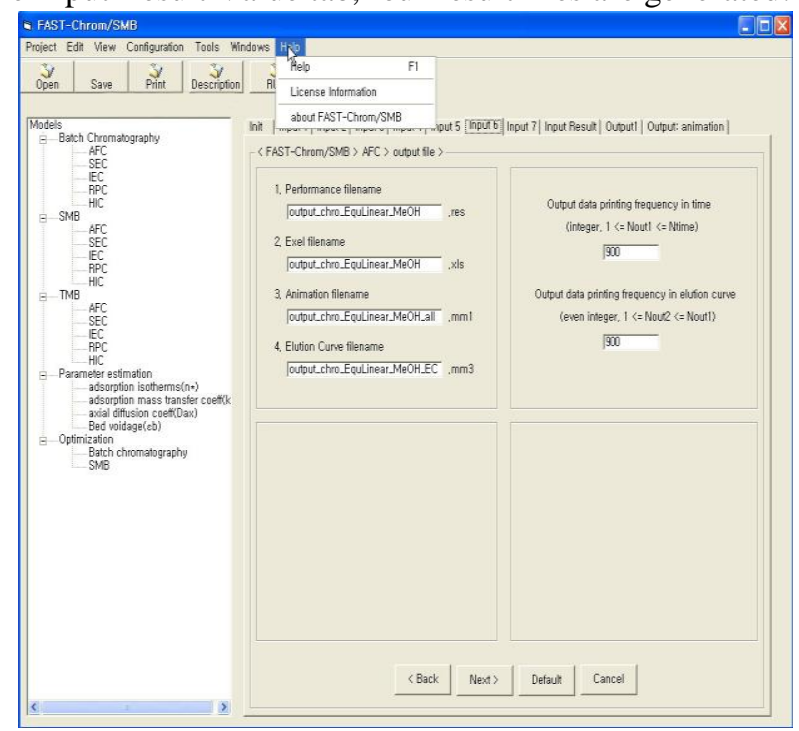

Fig. 6. Result File Creation Interface

Fig. 6 shows four result files generated by receiving input data for each step. Each item can be assigned a unique file name by the user, and the specified file is visualized as a result of the output simulation. Other items have the size of the file adjusted by allowing the user to specify the data frequency. The completed file appears as a graph and a movie in the Simulation tab.

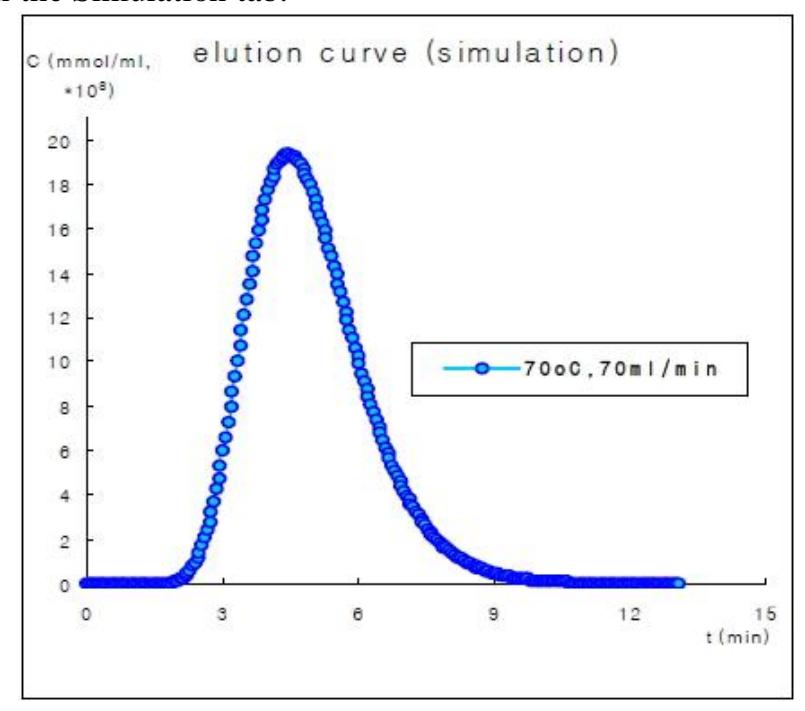

Fig. 7. Result Curve Graph

Fig. 7 shows two types of curve graphs that can be displayed on the Output Animation tab based on the four generated files. $70^{\circ} \mathrm{C}$, and $70 \mathrm{ml} / \mathrm{min}$, respectively. The result of this curve graph is very similar to the experimental data in real equipment. The overall error value was $0.26 \%$ and the accuracy was $99.74 \%$.

\section{CONCLUSION}

In this paper, a simulation system is constructed to enable visualization by inputting experimental data required for high performance adsorption process. In order to construct the simulation system, the internal engine uses the existing program, and the program is converted to be driven by switching parameters for compatibility. This allows visualization of the results through simulation without going through actual experiments. Thus, as the optimization of the process is added, time and cost can be saved significantly, and visualization of the numerical results improves performance. As a future work, the currently developed simulation system is limited to the adsorption process. Therefore, further development is needed to be able to apply various process methods in the future. And currently developed system visualizes the result value only in the data area where the input data is limited. In the future, you should improve the system performance version so that results can be obtained even in a large and large amount of experimental data input

\section{REFERENCES}

1. Alain Berthod, Mahmoud Hassoun. Journal of Chromatography A. 2006 May;1116(1):143-148.(Using the liquid nature of the stationary phase in countercurrent chromatography: IV. The cocurrent CCC method)

2. Xiaohai Han, Xiaolin Wei, Uwe Schnell, Klaus R.G. Hein. Combustion and Flame. Feb 2003;132(3):374-386.(Detailed modeling of hybrid reburn/SNCR processes for NOX reduction in coal-fired furnaces)

3. Martin Martinov, Dimiter Hadjiev, Serafim Vlaev. Process Biochemistry July 2010;45(7): 1023-1029.(Gas-liquid dispersion in a fibrous fixed bed biofilm reactor at growth and non-growth conditions)

4. S.S. Daood, M.T. Javed, B.M. Gibbs, W. Nimmo. Fuel. Mar 2013;105:283-292.(NOx control in coal combustion by combining biomass co-firing, oxygen enrichment and SNCR)

5. Muhammad Ayoub, Muhammad Faisal Irfan, Kyung-Seun Yoo. Energy Conversion and Management. Sep 2011;52(10): 3083-3088.(Surfactants as additives for NOx reduction during SNCR process with urea solution as reducing agent)

6. Jan Kloppenborg Møller, Henrik Madsen, Jacob Carstensen. Ecological Modelling. June 2011;222(11):1793-1799.(Parameter estimation in a simple stochastic differential equation for phytoplankton modelling)

7. M. Villegas, F. Augustin, A. Gilg, A. Hmaidi, U. Wever. Mathematics and Computers in Simulation. Jan 2012;82(5):805-817.(Application of the Polynomial Chaos Expansion to the simulation of chemical reactors with uncertainties)

8. Jake L. Rafferty, J. Ilja Siepmann, Mark R. Schure. Journal of Chromatography A. April 2011;1218(16):2203-2213.(Mobile phase effects in reversed-phase liquid chromatography: A comparison of acetonitrile/water and methanol/water solvents as studied by molecular simulation)

9. Melissa A. Holstein, Wai Keen Chung, Siddharth Parimal, Alexander S Freed, Blanca Barquera, Scott A. McCallum, Steven M. Cramer. Journal of Chromatography A. Mar 2012;1229:113-120.(Probing multimodal ligand binding regions on ubiquitin using nuclear magnetic resonance, chromatography, and molecular dynamics simulations)

10. Chen Wang, Jingming Hou, David Miller, Iain Brown and Yang Jiang International Journal of Disaster Risk Reduction. April 2019;35:150-165.(The role of integrated simulation and 3D visualization)

\section{AUTHORS PROFILE}

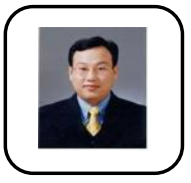

Byeong-Tae Ahn is a Faculty of Division of Liberal Arts at Anyang University, Korea. His research interests include: Image Processing, Video Analysis, IoT, BlockChain, Multimedia Database and MPEG-7. His address is: 37-22, Samduck Minahn-gu Anyang-City Gyeonggi-do, 430-714 South Korea. His phone number is +82-31-463-1204 and the email address is ahnbt@anyang.ac.kr. 